\title{
Bit Allocation and Encoding Parameter Selection for Rate-Controlled Error Resilient HEVC Video Encoding
}

\author{
Gosala Kulupana*, Dumidu S. Talagala*, Anil Fernando*, and Hemantha Kodikara Arachchi ${ }^{\$}$ \\ *Center for Vision, Speech and Signal Processing, University of Surrey, United Kingdom, \\ ${ }^{5}$ Department of Engineering, School of Science and Technology, Nottingham Trent University, United Kingdom

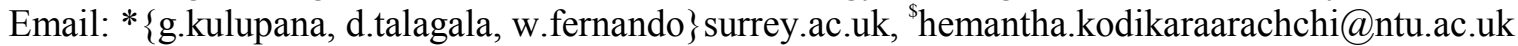

\begin{abstract}
Even though the latest video compression techniques such as High Efficiency Video coding (HEVC) have succeeded in significantly alleviating the bandwidth consumption during high resolution video transmission, they have become severely susceptible to transmission errors. Overcoming the resulting temporal impact of the transmission errors on the decoded video requires efficient error resilient schemes that can introduce robustness features to the coded video in order to mitigate the negative impact on the viewer. To this end, this paper proposes a rate-controlled error resilient bit allocation scheme, together with an encoding parameter selection process, to adaptively determine the most robust video coding parameters and the decoder error concealment operations during the encoding itself. Consequently, the proposed method has demonstrated 0.48dB-0.62dB PSNR gain over the state-of-the art methods at the same bit rate.
\end{abstract}

\section{INTRODUCTION}

Multimedia industry is getting bigger and bigger with the development of technologies that can produce and display closer to real-life video contents such as HD (High Definition) and $4 \mathrm{~K} / \mathrm{UHD}$ (Ultra High Definition) [1] formats. In the meantime, transmission of these enriched video contents has put forth numerous challenges to service providers attributed to their high bandwidth and storage requirements. The role of video compression technologies such as HEVC (High Efficiency Video Coding) is crucially important in this regard as higher levels of compression can reduce both the bandwidth and the storage requirements [2]. However, as a consequence of exploiting more and more spatial and temporal correlations to achieve greater compression efficiencies, the coded videos become significantly vulnerable to information losses in the generated bit stream, more so when the coded videos are transmitted over lossy channels such as the increasingly popular wireless mediums. Therefore, it is of utmost importance to improve the coded videos' robustness to errors prior to the transmission over error prone channels.

To this end, video error resiliency techniques are developed to address this issue by incorporating redundant information at the encoder, so that the lost information can be reconstructed to varying precisions at the decoder. In this work techniques are developed to achieve the error resiliency by adaptively integrating intra-coded blocks within the coded video such that any error due to a loss of information does not temporally propagate throughout the video sequence. The proposed method consists of two major novel components, (i) an HEVC based optimal bit allocation algorithm which predicts the number of bits prior to the encoding and (ii) robust encoding

This work was supported by the EU H2020 project CONTENT4ALL funded under H2020-INDUSTRIAL LEADERSHIP program. (Project ID: 762021). parameters derivation process which includes motion vectors, coding modes and the error concealment type.

The remainder of this paper is organized as follows. First, a discussion of the state-of-the art in video error resilience is presented in Section II. This is followed by the proposed methodology in Section III. The performance of the proposed method is evaluated in Section IV, and is followed by the concluding remarks in Section V.

\section{RELATED WORK}

A complete video error resilient framework comprises of two major inter-related operations namely error resilient video compression at the encoder and error concealment at the decoder. Out of all the available error concealment techniques, slice-copying and motion-copying are widely used in practical applications and both have been incorporated in this work consistent with the video content.

Moving to the error resilient aspects, Zhang et al. in [3] and Zhihai et al. in [4] have modeled the overall distortion of an H.263 coded video in terms of the quantization distortion and the channel distortion, assuming motion-copying and slicecopying respectively at the decoder. Later the distortion terms are re-used inside the rate-distortion cost function to determine the best coding mode. The authors in [5] extended the same model to H.264 and incorporated the error propagation impact to the motion estimation process.

However, these resiliency schemes cannot be immediately applied to the HEVC framework in their original form. The stronger temporal correlation in HEVC compared to H.264, leads to severe error propagation in the temporal domain [6] which cannot be captured by conventional error propagation models. In [7], the authors have investigated the effect of packet loss, latency, bandwidth on the perceived video quality for an HEVC coded video. Furthermore, a temporal domain error propagation analysis and mitigation approach was presented in [8], and a similar approach which effectively switches the TMVP feature in HEVC has been presented in [9]. However, these error resilient schemes only investigate the effect of error propagation due to motion vectors, and pixel domain error propagation has therefore not been considered. Furthermore, none of these methods have considered the optimal bit allocation during a rate-controlled scenario.

To this end, this paper proposes an error resilient framework for HEVC codec which operates in line with the HEVC rate controller. The proposed scheme comprises of two major novel components: an optimal bit allocation framework operating under erroneous environments prior to the encoding and a prediction model which derives the most robust encoding parameters and concealment operations during the encoding. 


\section{PRoposed Method}

Fig.1 illustrates the architecture of the proposed error resilient framework which comprises of three major components. The first component is accountable for optimal target bit allocation under a rate-controlled environment prior to the encoding whereas the second and the third components are designed to derive the most robust motion vectors and other video coding parameters for the derived target bit rate prior to the transmission over an error prone channel.

\section{A. Error Resilient Motion Vector Estimation}

Motion vector estimation inside the standard HEVC HM [10] encoder is performed by minimizing the cost function,

$$
J_{\text {motion }}=S A D_{\text {no_error }}+\lambda_{\text {motion }} \cdot R_{m v}
$$

where $S A D_{\text {no_error }}=\sum_{\forall i}\left|X_{n}^{i}-\hat{X}_{n-1}^{j}\right|, \lambda_{\text {motion }}=\sqrt{\lambda_{\text {mode }}}$ is

the Lagrangian multiplier which balances the rate cost and the residual cost during motion estimation and $R_{m v}$ is the rate incurred by motion vectors. (All the notations used in this work are listed in Table I).

However, the impact of the selected motion vectors on the propagated error from the reference picture is not accounted in (1). Therefore, the motion estimation cost function under erroneous environments is amended as,

$$
J_{\text {motion }}^{\prime}=S A D_{\text {error }}+\lambda_{\text {motion }} \cdot R_{m v}
$$

Where, $S A D_{\text {error }}=\sum_{\forall i}\left|E\left\{X_{n}^{i}-\tilde{X}_{n-1}^{j}\right\}\right|$

$$
\begin{aligned}
& =\sum_{\forall i}\left|X_{n}^{i}-\hat{X}_{n-1}^{j}+\hat{X}_{n-1}^{j}-\tilde{X}_{n-1}^{j}\right| \\
& =\sum_{\forall i}\left|X_{n}^{i}-\hat{X}_{n-1}^{j}+E P_{D}\left\{\hat{X}_{n-1}^{j}\right\}\right|
\end{aligned}
$$

In (3), $E P_{D}\left\{\hat{X}_{n-1}^{j}\right\}$ is the pixel level channel error propagation term which can be further simplified as,

$$
\begin{aligned}
& E P_{D}\left\{\hat{X}_{n-1}^{j}\right\}=E\left\{\hat{X}_{n-1}^{j}-\widetilde{X}_{n-1}^{j}\right\} \\
& =p \cdot E C_{D}\left\{\hat{X}_{n-1}^{j}\right\}+p \cdot E P_{D}\left\{\hat{X}_{n-2}^{m}\right\}+(1-p) \cdot E P_{D}\left\{\hat{X}_{n-2}^{k}\right\}
\end{aligned}
$$

Here $E C_{D}\left\{\hat{X}_{n-1}^{j}\right\}=\hat{X}_{n-1}^{j}-\hat{X}_{n-2}^{u}$ is the concealment error.

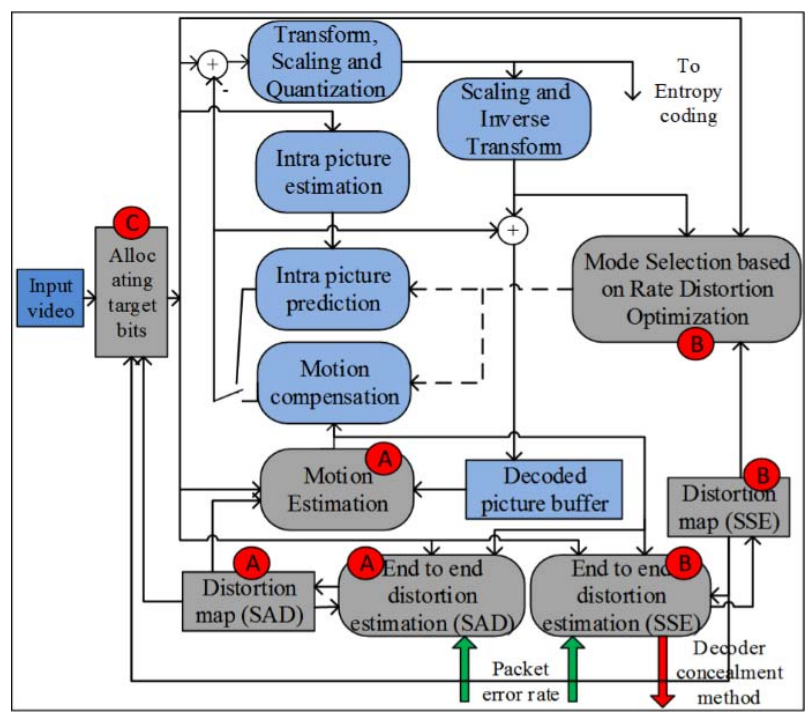

Fig. 1. Proposed error resilient HEVC encoder
TABLE I

TERMS AND NOTATIONS

Term

Notation

Currently encoding frame number

$n^{\text {th }}$ frame of the video sequence

Location of the currently encoding pixel

Packet error rate

Original pixel $i$ of frame $n$

Reconstructed pixel $i$ of frame $n$ at the encoder

Decoded pixel $i$ of frame $n$ after going through a lossy channel

Residual of pixel $i$ of frame $n$

Location of the reference pixel of the pixel $i$ of frame $n$

Location of the reference pixel of the pixel $j$ of frame $n-1$

Location of the concealment pixel of the pixel $i$ of frame $n$

Location of the concealment pixel of the pixel $j$ of frame $n-1$

Location of the motion compensated pixel of the pixel $i$ of frame $n$

Expected value operator

Operator for pixel level channel propagation error

Operator for pixel level channel concealment error

Operator for pixel level squared channel propagation error

Operator for pixel level squared channel concealment error

\section{B. Error Resilient Aware Mode Selection}

After deriving the motion vectors as explained in the previous sub section, the standard HM encoder determines the rest of the coding parameters (i.e., coding structure, coding mode, quantization parameter) by minimizing,

$$
J_{\text {mode }}=S S E_{\text {no_error }}+\lambda_{\text {mode }} \cdot\left(R_{m v}+R_{\text {residual }}\right)
$$

where $\quad S S E_{n o \_ \text {error }}=\sum_{\forall i}\left(X_{n}^{i}-\hat{X}_{n}^{i}\right)^{2}$ and $\lambda_{\text {mode }}$ is the Lagrangian parameter which trades-off the bit rate cost to that of the quantization distortion and is derived according to the allocated bit rate for the particular block. $R_{\text {residual }}$ is the rate incurred during the quantized residual transmission.

Yet again, the cost function in (4) does not evaluate the impact of the error propagation from the reference picture and therefore is amended in this work as below,

$$
J_{\text {mode }}^{\prime}=S S E_{\text {error }}+\lambda_{\text {mode }} \cdot\left(R_{m v}+R_{\text {residual }}\right)
$$

where $S S E_{\text {error }}=\sum_{\forall i} E\left\{X_{n}^{i}-\tilde{X}_{n}^{i}\right\}^{2}$

$=\sum_{\forall i}\left(X_{n}^{i}-\hat{X}_{n}^{i}\right)^{2}+E\left\{\hat{X}_{n}^{i}-\tilde{X}_{n}^{i}\right\}^{2}+2 .\left(X_{n}^{i}-\hat{X}_{n}^{i}\right) E\left\{\hat{X}_{n}^{i}-\tilde{X}_{n}^{i}\right\}$ $=S S E_{n o \_ \text {error }}+\sum_{\forall i} E\left\{\hat{X}_{n}^{i}-\tilde{X}_{n}^{i}\right\}^{2}+2 .\left(X_{n}^{i}-\hat{X}_{n}^{i}\right) E P_{D}\left\{\hat{X}_{n}^{i}\right\}$

Here the squared channel error term $E\left\{\hat{X}_{n}^{i}-\widetilde{X}_{n}^{i}\right\}^{2}$ can be iteratively calculated as,

$$
\begin{aligned}
= & (1-p) \cdot E\left\{\hat{X}_{n-1}^{j}+r_{n}^{i}-\tilde{X}_{n-1}^{j}-r_{n}^{i}\right\}^{2}+p \cdot E\left\{\hat{X}_{n}^{i}-\widetilde{X}_{n-1}^{u}\right\}^{2} \\
= & (1-p) \cdot E\left\{\hat{X}_{n-1}^{j}-\tilde{X}_{n-1}^{j}\right\}^{2}+p \cdot E\left\{\hat{X}_{n}^{i}-\hat{X}_{n-1}^{u}+\hat{X}_{n-1}^{u}-\tilde{X}_{n-1}^{u}\right\}^{2} \\
= & (1-p) \cdot E P_{S E}\left\{\hat{X}_{n-1}^{j}\right\}+p \cdot\left(\hat{X}_{n}^{i}-\hat{X}_{n-1}^{u}\right)^{2} \\
& \quad+p \cdot E\left\{\hat{X}_{n-1}^{u}-\tilde{X}_{n-1}^{u}\right\}^{2}+p \cdot 2 \cdot E\left\{\hat{X}_{n-1}^{u}-\tilde{X}_{n-1}^{u}\right\}\left(\hat{X}_{n}^{i}-\hat{X}_{n-1}^{u}\right) \\
= & (1-p) \cdot E P_{S E}\left\{\hat{X}_{n-1}^{j}\right\}+p \cdot E C_{S E}\left\{\hat{X}_{n}^{i}\right\} \\
& \quad+p \cdot E P_{S E}\left\{\hat{X}_{n-1}^{u}\right\}+2 \cdot p \cdot E P_{D}\left\{\hat{X}_{n-1}^{u}\right\} \cdot E C_{D}\left\{\hat{X}_{n}^{i}\right\}
\end{aligned}
$$


Here $E P_{S E}\left\{\hat{X}_{n-1}^{j}\right\}=E\left\{\hat{X}_{n-1}^{j}-\tilde{X}_{n-1}^{j}\right\}^{2}$ is the squared error

propagation term whereas the squared error concealment term becomes $E C_{S E}\left\{\hat{X}_{n}^{i}\right\}=\left(\hat{X}_{n}^{i}-\hat{X}_{n-1}^{h}\right)^{2}$. It should be noted that the last term in (6) is decomposable in above form as $E C_{D}$ being a constant term, is independent of the loss probability. Furthermore, the first term in (6) vanishes for intra blocks.

Equation (6) is computed by evaluating the proposed adaptive error concealment scheme in Condition 1 which is evaluated per slice basis. The chosen concealment mode is subsequently transmitted separately as an SEI (Supplemental Enhancement Information) NAL unit available in HEVC. The decoder uses this information while reconstructing a lost slice.

\section{Optimal Target Bit Allocation}

The standard HEVC HM encoder is designed in such a way that first the total available bits are distributed among the GOPs (Group of Pictures) and later the bits within a GOP are distributed among the video pictures and finally the bits within a picture are distributed among the CTUs (Coding Tree Units) [11]. While distributing the bits among the GOPs, the standard procedure devises a sliding window based mechanism which accounts for the discrepancies between the allocated and the consumed bits in the previous GOP. Similarly, in order to distribute the bits among the pictures within a GOP, HM encoder employs a hierarchical bit allocation scheme with predefined weights for each picture level. Finally, CTU level bit allocation is performed based on the allocated and the consumed bits of the co-located CTU in the reference frame.

During the CTU level bit allocation under erroneous environments Algorithm 1 is integrated in place of the standard bit allocation. As indicated in the Algorithm 1, the bit allocation for the current CTU is done based on the error propagation from the co-located CTU in the reference frame. Consequently, when the error propagation from the previous CTU is significantly higher than the rest of the CTUs within the frame, current CTU is allocated with significantly larger number of bits which may eventually be sufficient to encode the current block in intra-mode and to terminate the error propagation.

\section{RESULTS AND DISCUSSION}

The proposed error resilient framework and two reference methods [4], [5] are implemented in the HM16.2 HEVC reference software [10]. The configuration parameters used during the simulations are summarized in Table II. The CTUs (Coding Tree Units) within a frame are grouped into slices such that each slice contains a single row of CTUs during

Condition 1
if $\left(\sum_{i \in \text { slice }}\left(\hat{X}_{n}^{i}-\hat{X}_{n-1}^{v}\right)^{2}+E P_{S E}\left\{\hat{X}_{n-1}^{v}\right\}<\right.$
$\left.\qquad \sum_{i \in \text { slice }}\left(\hat{X}_{n}^{i}-\hat{X}_{n-1}^{i}\right)^{2}+E P_{S E}\left\{\hat{X}_{n-1}^{i}\right\}\right)$
$\quad$ Choose motion copying as the concealment method.
$\quad$ Update the distortion calculation with $u=v ;$
else
$\quad$ Choose slice copying. Make $u=i ;$

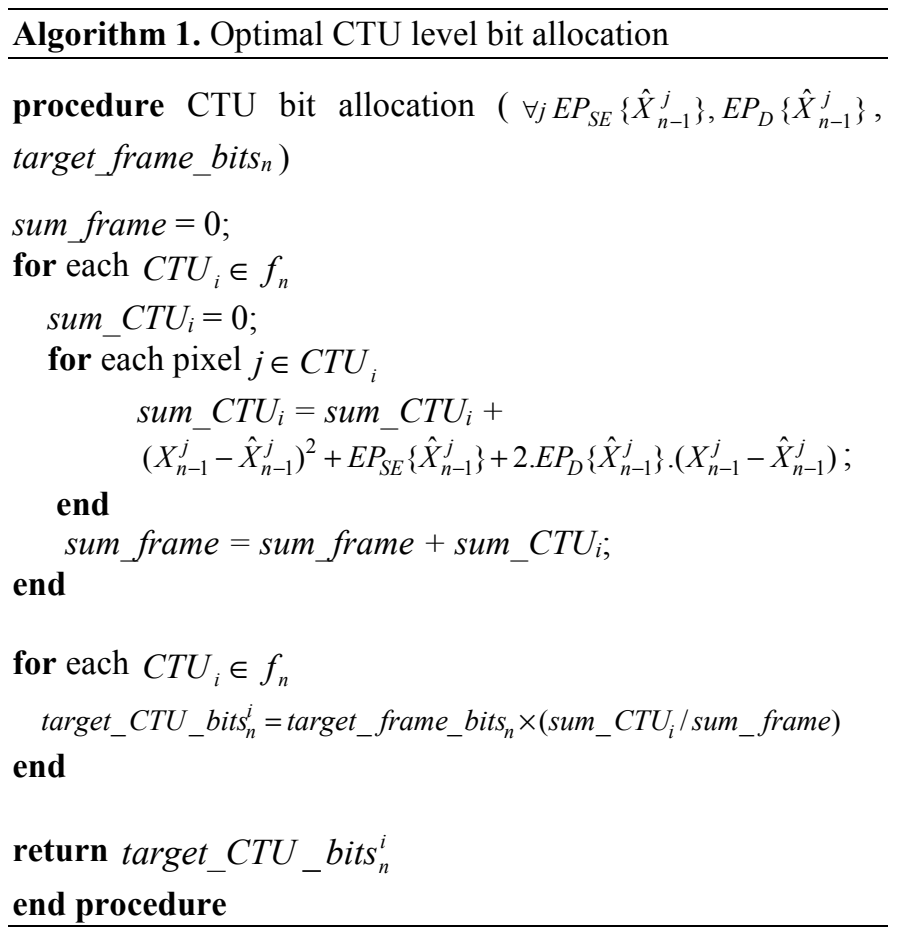

the error resiliency inclusion. These values can be found in Table II. The bit rates for different resolutions are chosen such that each sequence has an acceptable level of perceptual quality. (i.e., the chosen bit rates for HD videos: 8Mbps, Park

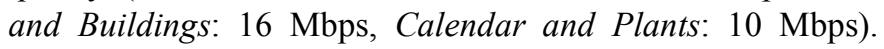
Finally, each coded video is evaluated for objective video quality after concealment operation at the decoder for $2 \%, 5 \%$, $10 \%$ and $15 \%$ random packet drops.

Table III summarizes the PSNR based objective video quality results for the proposed method, two state-of-the-art methods namely Zhihai [4] and $\mathrm{Md}+\mathrm{Me}$ [5] and the standard HM encoding with motion-copying and slice-copying concealments at the decoder. The results are compared for six different video sequences. As, it can be seen in Table III, the proposed method has outperformed the state-of-the-art methods with a significant margin for all the packet error rates considered. The gains of the proposed method under different packet error rates have demonstrated similar performances leading to the conclusion that the proposed method can be equally applied in different forms of network conditions (i.e.,

TABLE II

HEVC SIMULATION PARAMETERS

\begin{tabular}{lc}
\hline \multicolumn{1}{c}{ Configuration parameter } & Values \\
\hline GOP (Group of Picture) size & 4 \\
Number of Encoded frames (HD) & 100 \\
Number of Encoded frames (UHD) & 50 \\
Frame Rate & $25 \mathrm{~Hz}$ \\
Bit depth & 8 \\
HEVC configuration & encoder_lowdelay_P_main \\
HEVC profile & main \\
Rate control method & CTU level rate control \\
Initial QP & 30 \\
Number of CTUs per slice (HD) & 30 \\
Number of CTUs per slice (UHD) & 60 \\
\hline \hline
\end{tabular}


not particularly lossy channels to extremely lossy channels). In the meantime, as expected the state-of-the-art error resilient schemes have beaten the HM based concealment only approaches. Furthermore, the relative gain of the UHD content has become more visible compared to the HD content which can be interpreted as the accuracy of the coding parameter selection process becomes more crucial when there is a large amount of information, for instance the UHD videos contents.

Fig.2 illustrates the behaviors of the resilient schemes under different bit rates for Musicians video sequence at $10 \%$ packet error rate (PER). As it is evident, the proposed optimal bit allocating algorithm has become more informative while allocating bit rates specially at lower bit rates making it ideal for low bit rate applications.

TABLE III

PSNR RESULTS FOR DIFFERENT SEQUENCES (ALL IN dB)

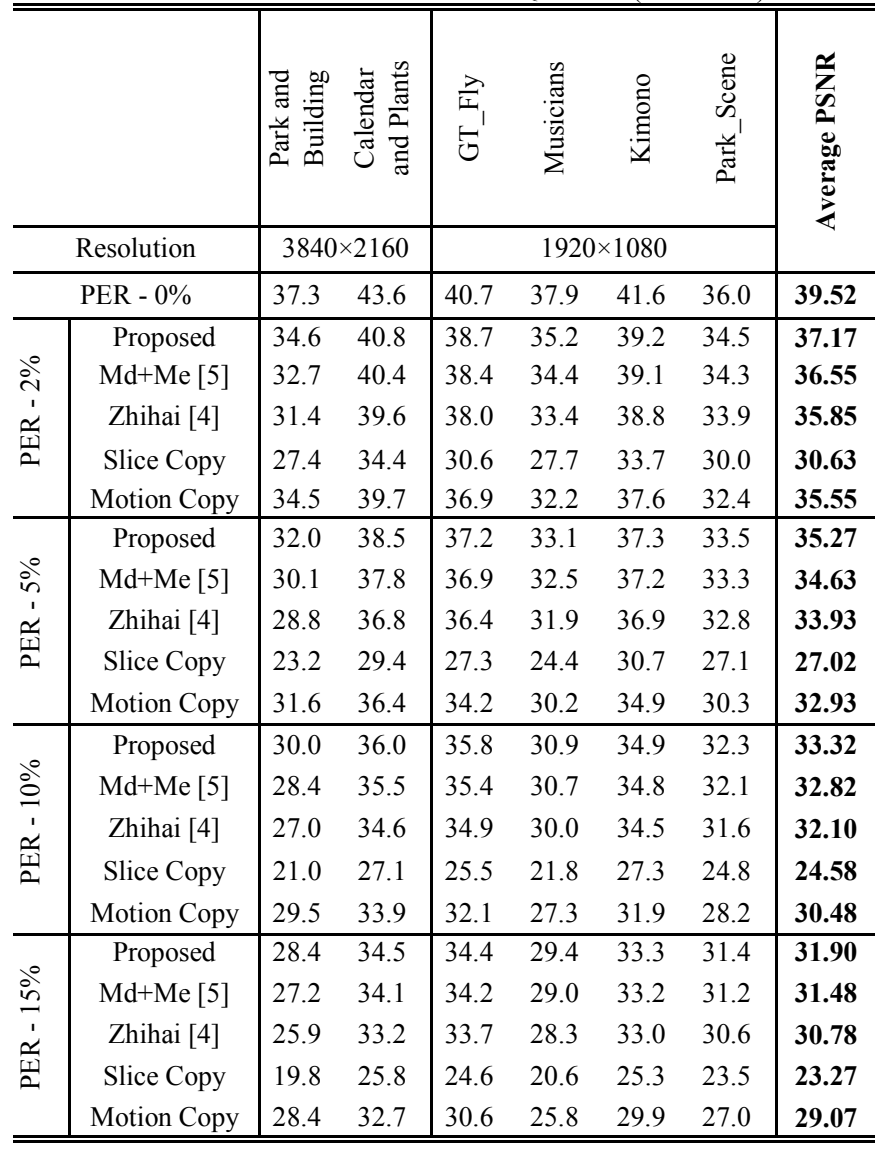

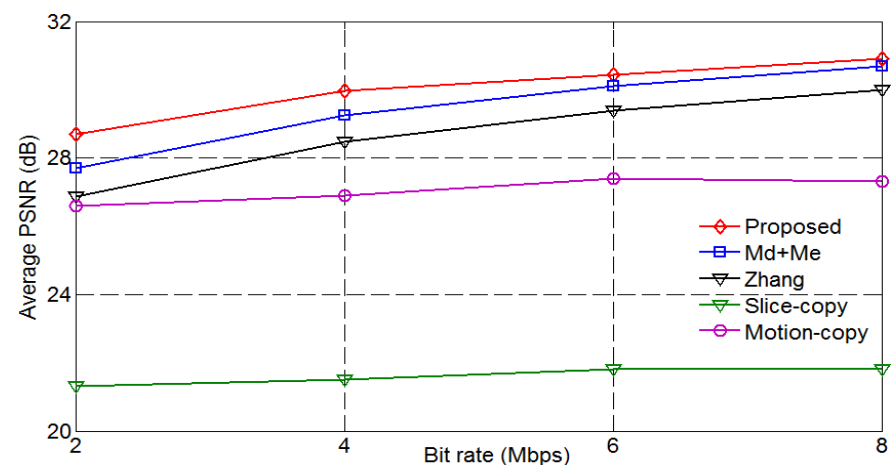

[10]

\section{CONCLUSION}

In this paper, a novel error resilient scheme compatible with the HEVC codec was presented. The proposed method consists of two novel components namely the optimal target bit allocation under erroneous environments and content adaptive coding parameter selection which includes most robust coding modes, motion vectors, coding structures as well as the optimal concealment operation at the decoder. The proposed resiliency scheme is compared against two state-of-the-art error resilient schemes and the standard HM based HEVC encoding [10] and has demonstrated an average $0.48-0.62 \mathrm{~dB}$ performance improvement over the state-of-the art methods and up to $2.5 \mathrm{~dB}$ gain over the standard HM encoder. Future work involves modeling the R-D behavior for error resilient HEVC encoding.

\section{REFERENCES}

Fig. 2 PSNR values for different bit rates Musicians @ 10\% PER
E. Nakasu, "Super Hi-Vision on the Horizon: A Future TV System That Conveys an Enhanced Sense of Reality and Presence," IEEE Consum. Electron. Mag., vol. 1, no. 2, pp. 36-42, Apr. 2012.

T. K. Tan, R. Weerakkody, M. Mrak, N. Ramzan, V. Baroncini, J.R. Ohm, and G. J. Sullivan, "Video Quality Evaluation Methodology and Verification Testing of HEVC Compression Performance," IEEE Trans. Circuits Syst. Video Technol., vol. 26, no. 1, pp. 76-90, Jan. 2016.

Zhang Rui, Shankar L. Regunathan, and Kenneth Rose. "Video coding with optimal inter/intra-mode switching for packet loss resilience." IEEE Journal on Selected Areas in Commun., vol. 18 no. 6, pp. 966-976, Jun. 2000.

Zhihai He, Jianfei Cai, and Chang Wen Chen, "Joint source channel rate-distortion analysis for adaptive mode selection and rate control in wireless video coding," IEEE Trans. Circuits Syst. Video Technol., vol. 12, no. 6, pp. 511-523, Jun. 2002.

C.-H. Lu and Y. Lin, "Robust error resilient H.264/AVC video coding," in Proc. IEEE Int. Region 10 Conf., Xi'an, Oct. 2013, pp. $1-4$.

B. Oztas and M. Pourazad, "A study on the HEVC performance over lossy networks," in Proc. IEEE Int. Conf. Electron. Circuits Syst., Seville, Dec. 2012, pp. 785-788.

J. Nightingale, Q. Wang, and C. Grecos, "HEVStream: a framework for streaming and evaluation of high efficiency video coding (HEVC) content in loss-prone networks," IEEE Trans. Consum. Electron., vol. 58, no. 2, pp. 404-412, July 2012.

B. Li, J. Xu, and H. Li, "Parsing robustness in high efficiency video Communications and Image Processing, Tainan, Nov. 2011, pp. 14.

J. Carreira, V. De Silva, E. Ekmekcioglu, A. Kondoz, P. Assuncao, and S. Faria, "Dynamic motion vector refreshing for enhanced error resilience in HEVC." in Proc. IEEE 22nd European Signal Processing Conf., Lisbon, Sept. 2014, pp. 281-285.

"HM software manual." [Online]. Available: https://hevc.hhi.fraunhofer.de/svn/svn_HEVCSoftware/.

B. Li, H. Li, L. Li, and J. Zhang, “ $\lambda$ Domain Rate Control Algorithm for High Efficiency Video Coding," IEEE Trans. Image Process., vol. 23, no. 9, pp. 3841-3854, Sep. 2014. coding-analysis and improvement," in Proc. IEEE Conf. Visual 